\title{
LOW LOSS RF MEMS PHASE SHIFTERS FOR SATELLITE COMMUNICATION SYSTEMS
}

Guan-Leng Tan and Gabriel Rebeiz (University of Michigan, Ann Arbor, MI), Robert Mihailovich and Jeffrey DeNatale (Rockwell Scientific Company, Thousand Oaks, CA), William J. Taft and Nafiz Karabudak (Lockheed Martin Commercial Space Systems, Newtown, PA), William P. Kornrumpf (GE Corporate R\&D, Schenectady, NY)

\begin{abstract}
$\underline{\text { ABSTRACT }}$
RF MEMS represents an attractive enabling technology for future satellite communications systems. In particular, MEMS RF switch devices are characterized by low insertion loss, low power consumption, and minimal weight and volume, offering significant systemlevel benefits to space-based applications. The mechanical operation of the MEMS devices is expected to result in improved stability in radiation environments relative to semiconductor devices. A number of circuit applications of the MEMS technology have been explored, such as MEMS-based phase shifter circuits for electronically scanned antennas. These phase shifter concepts have been demonstrated and shown to provide unique capabilities with respect to loss, bandwidth, size and weight relative to competing technologies.
\end{abstract}

A novel low-loss phase shifter, based on RF MEMS series switches and a single-pole four-throw (SP4T) switch design, is presented. The phase shifter is fabricated on a $200 \mu$ m-thick GaAs substrate, and occupies less than $12 \mathrm{~mm}^{2}$ of space. The measured average insertion loss is $-0.55 \mathrm{~dB}$, with a reflection loss of less than $-17 \mathrm{~dB}$ over the $8-12 \mathrm{GHz}$ frequency range. The 2-bit phase shifter performs well up to $18 \mathrm{GHz}$ with an average loss of only $-0.85 \mathrm{~dB}$ and a near-perfect linear phase shift from DC-18 GHz. This is the lowest loss MMIC-type phase shifter to-date at 8-18 GHz.

\section{INTRODUCTION}

RF MEMS phase shifters have been developed using capacitive or DC-contact MEMS switches [1, 2, 3, 4]. They are characterized by near-zero power consumption and low-loss performance. The MEMS phase shifters are based on traditional switched-line designs using singlepole double-throw switches [1,3] or reflect-line designs with 3-dB couplers [2] or on switched distributed MEMS transmission-lines [4]. Typical X-band 2-bit phase shifters show a loss of -0.9 to $-1.0 \mathrm{~dB}$ when integrated on a GaAs or a high-resistivity silicon substrate, although Goldsmith et al. attained an average loss of $-0.65 \mathrm{~dB}$ at
8.5 $\mathrm{GHz}$ by integrating the 3 - $\mathrm{dB}$ couplers on a ceramic substrate. This results in a hybrid-type phase shifter, with increased assembly cost.

The novel design presented in this paper is based on the realization that one can build a very compact, highisolation single-pole four-throw switch using RF MEMS DC-contact series switches with a very low up-state capacitance. The SP4T switch can be well-matched up to $18 \mathrm{GHz}$ using a short inductive transmission line. A 2-bit phase shifter can therefore be synthesized using two SP4T switches, with 4 different sections of transmission lines and lengths of $0,90,180$ and $270^{\circ}$, respectively (Fig. 1a). The advantage is that the RF energy passes by two switches instead of four as in a standard switchedline design. Another advantage is that if a bit is not used, the energy does not pass by a "dead" section of transmission-line (Fig. 1b). Compared to reflect-line designs, the SP4T approach does not use a 3-dB coupler and therefore does not suffer from the associated roundtrip loss.
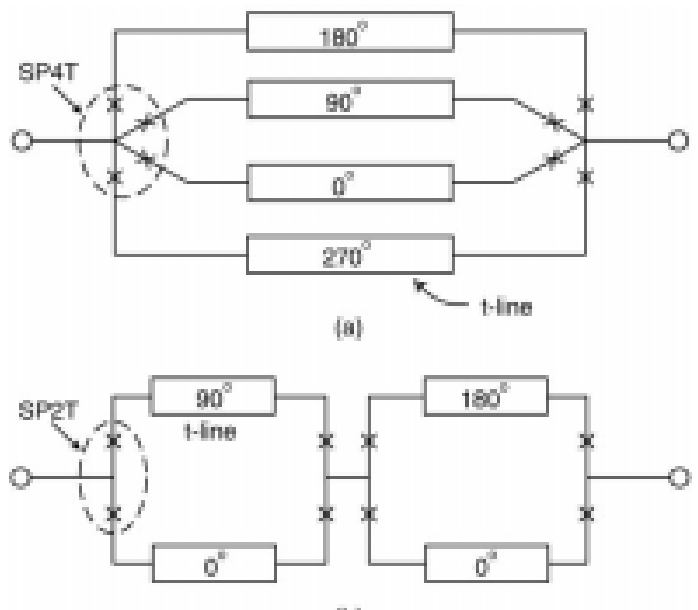

(b)

Fig. 1 Two-bit phase shifters based on the (a) SP4T, and (b) SP2T, approach 


\section{DESIGN AND MEASUREMENTS}

The design of the SP4T phase shifter is carried out with a three-step approach. First, the SP4T switch is matched using short inductive sections and Agilent ADS/Momentum, and results in excellent performance up to $20 \mathrm{GHz}$. Second, the four delay lines are simulated using Agilent Momentum as an 8-port network. The resulting S-parameter matrix includes the coupling between the lines and allows us to change the delay line geometry to yield the most compact layout. After several iterations, the SP4T switches are cascaded with the delay lines to yield the overall performance of the phase shifter. Notice that two short open-stubs are placed in the $270^{\circ}$ delay line for improved matching in the $8-12 \mathrm{GHz}$ band.

The SP4T is fabricated at Rockwell Scientific on a 200 $\mu \mathrm{m}$ GaAs substrate using an RF MEMS series switch described in detail in [5]. The Rockwell Scientific switch has a $\mathrm{C}_{\mathrm{u}}$ of $2 \mathrm{fF}$ and an isolation of $-38 \mathrm{~dB}$ at $10 \mathrm{GHz}$. A gold-alloy contact metal is used, and the average downstate switch resistance is $1 \Omega$. Via-holes around the switches are used for DC-biasing, i.e. ground return (Fig. 1a). The overall area of the chip including the biasing network is $12 \mathrm{~mm}^{2}$, making this the smallest RF MEMS phase shifter to-date. A photograph of the fabricated phase shifter is shown in Fig. 2.

The measurements are calibrated to the probe tip and include the loss of the input and output CPW-tomicrostrip transitions. An average loss of -0.5 to $-0.6 \mathrm{~dB}$ at $8-12 \mathrm{GHz}$ is obtained, with a reflection loss of better than $-17 \mathrm{~dB}$ in all four states (Fig. 3). The measured differential time delays are $0,23.0,49.0$ and 72.8 ps respectively, which correspond to a differential phase shift of $0,90.1,177.8$ and $272.0^{\circ}$ at $10.25 \mathrm{GHz}$ (Fig. 4). The measured phase shift is linear up to $18 \mathrm{GHz}$, making this design a true time delay phase shifter from 10-18 $\mathrm{GHz}$. At $18 \mathrm{GHz}$, the measured average loss is only $0.85 \mathrm{~dB}$, with a reflection loss of better than $-11 \mathrm{~dB}$. ADS/Momentum simulations agree very well with the measurements.

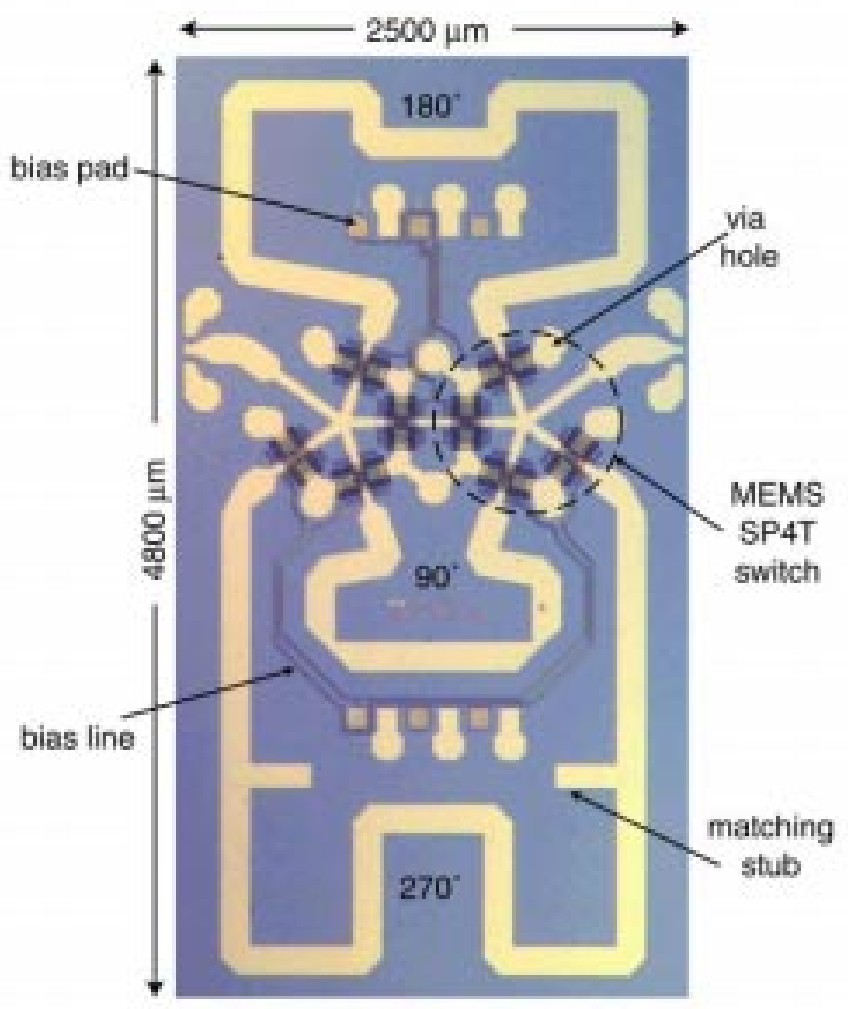

Fig. 2 Photograph of the low-loss SP4T 2-bit phase shifter. 

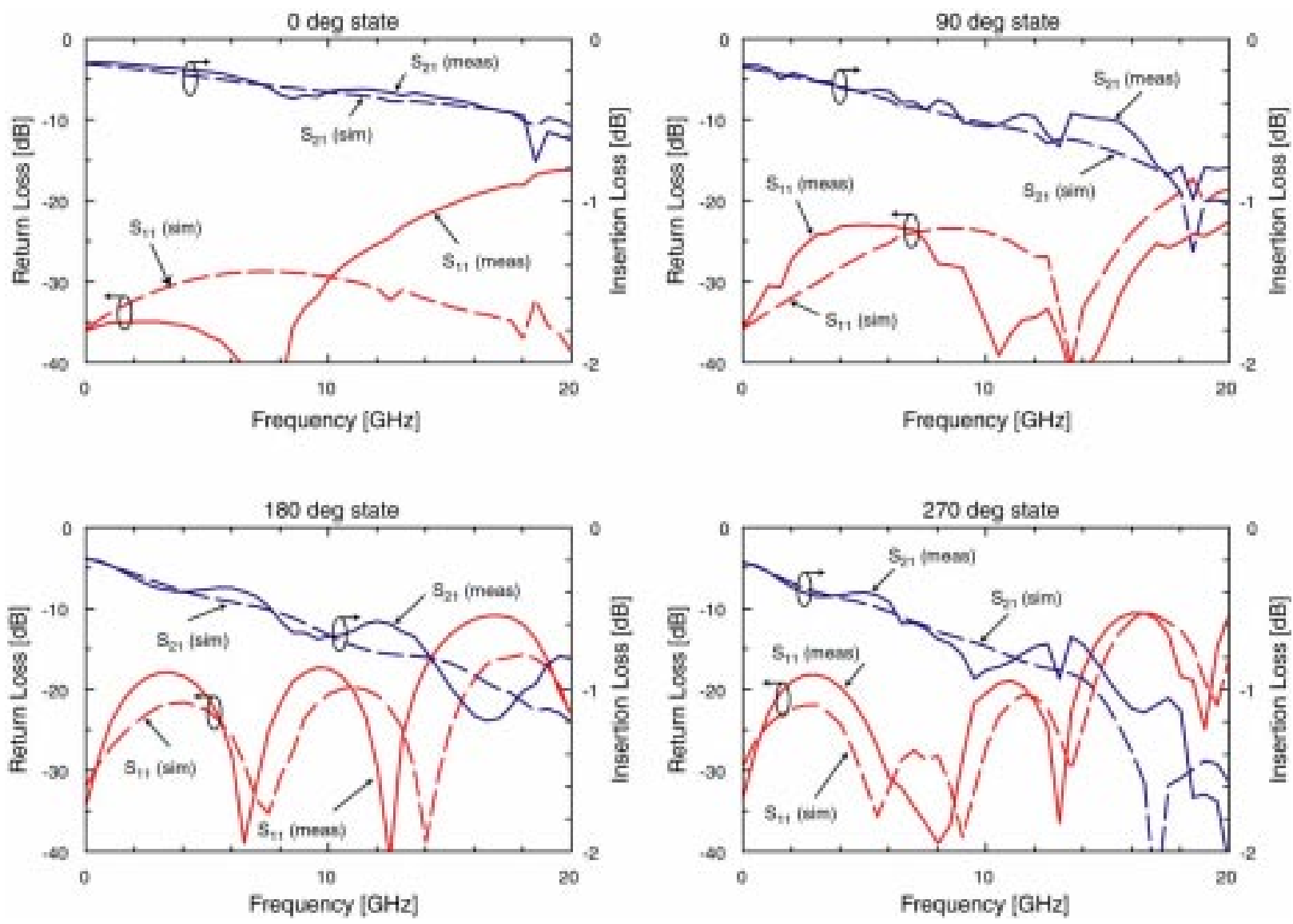

Fig. 3 Measured insertion loss and reflection loss of the SP4T 2-bit phase shifter (theory and experiment).
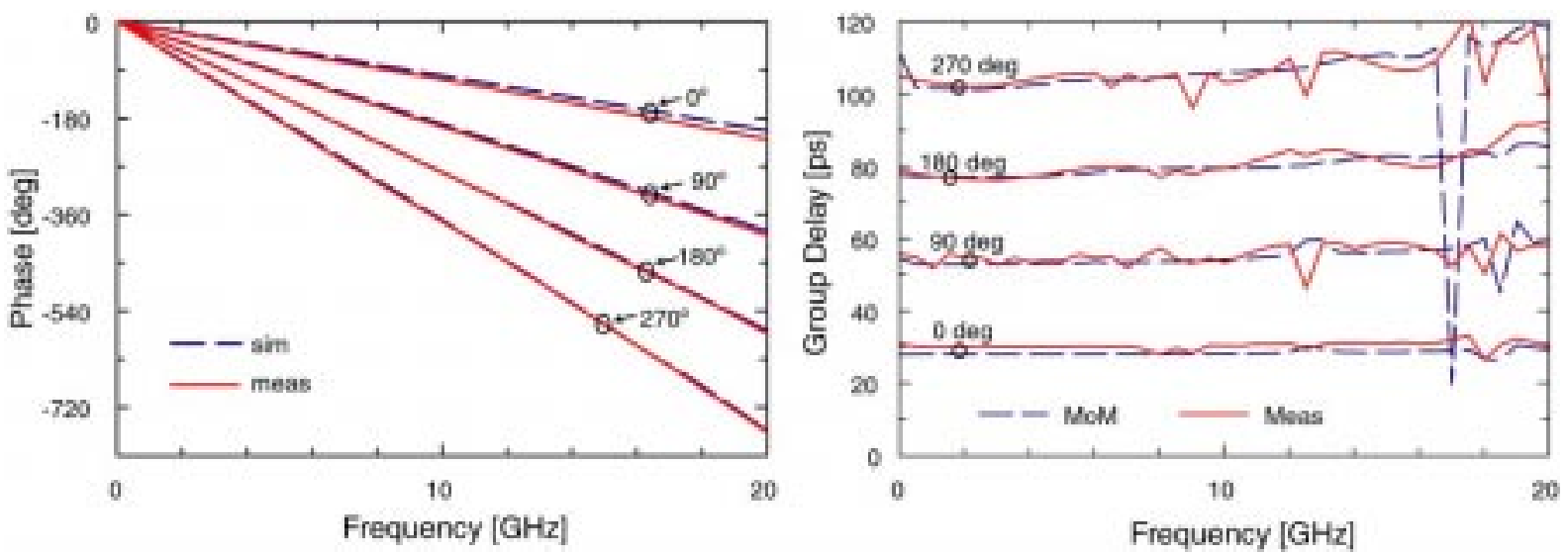

Fig. 4 Measured phase shift and group delay of the SP4T 2-bit phase shifter (theory and experiment). 


\section{EXTENSION TO 4-BIT DESIGNS}

The SP4T design can be extended to 4-bit phase shifters as shown in Fig. 5. In this case, a $0 / 22.5 / 45 / 67.5^{\circ}$-cell is cascaded after the 2-bit phase shifter. Again, the RF energy passes by 4 switches instead of 8 as in a standard switched-line design. This results in at least $0.4 \mathrm{~dB}$ less loss. The phase shifter area including biasing networks is $21 \mathrm{~mm}^{2}$ on a 200 $\mu \mathrm{m}$-thick GaAs substrate. Simulations indicate an average loss of $-0.85 \mathrm{~dB}$, with a reflection loss below $-18 \mathrm{~dB}$ at $10 \mathrm{GHz}$, and a linear phase-shift up to 18 GHz. Measurements will be presented at the conference.

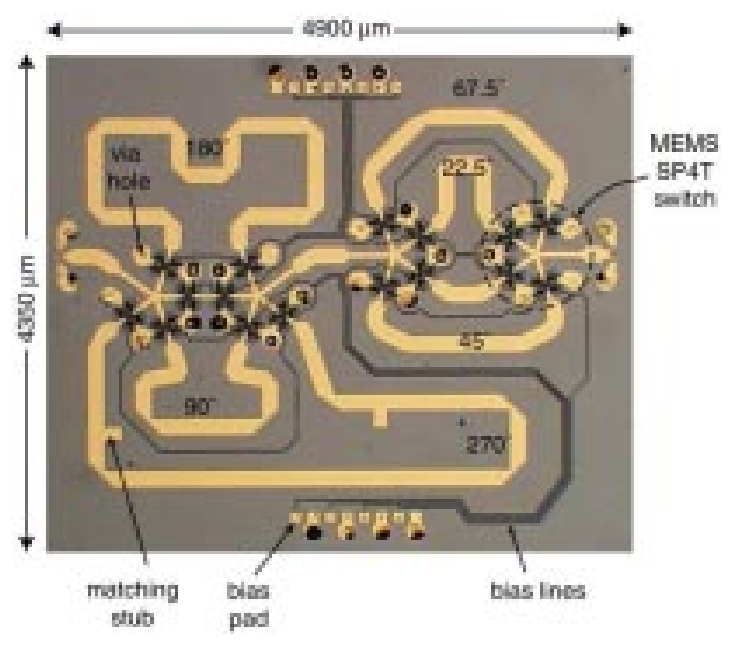

Fig. 5 Photograph of a 4-bit SP4T-based phase shifter.

\section{PACKAGING APPROACH}

To meet the challenges of MEMS packaging, CSP packaging approach which is an extension of the Lockheed Martin/GE High Density Interconnect $[6,7,8]$ process is studied. Chip Scale Packaging approach (CSP) has been used to package microwave devices such as GaAs MMICs that have sensitive mechanical structures similar to MEMS cantilevers. These sensitive structures are the air bridges used to provide low capacitance crossovers for the RF and DC signal. The process flow for packaging MEMS devices is similar to the MMIC process with the exception that the cavities are larger and deeper. Figure 6 is a conceptual cross section of the packaging approach selected.

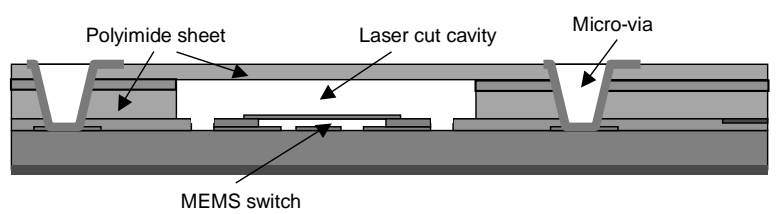

Fig. 6. MEMS packaging approach based on Polymeric Chip-on-Flex technology.

In this approach, a sheet of polyimide is first perforated with cavities at the location of each MEMS switch and coated with a thermoset adhesive. [9] The MEMS phase shifters are then bonded to the face of the polyimide using a precision pick and place robot with both up and down facing alignment cameras so that the MEMS devices fall precisely in the preformed cavities. After bonding the top layer is applied and the entire stack is cured in a vacuum oven. Microvias are then laser drilled to the MEMS phase shifter bond pads, metal is deposited in the microvias and patterned to provide wirebondable connection points on the surface of the CSP. While figure 6 shows a single MEMS phase shifter for clarity, in practice, it is possible to package a number of phase shifters at one time by bonding a complete reticule or larger part of a wafer. Figure 7 is a photograph of a reticule of the 2 bit phase shifter as bonded to the polyimide sheet. Figure 8 is a close up of the cavities formed in the polyimide and demonstrates the ability to accurately align the MEMS features to the cavities.

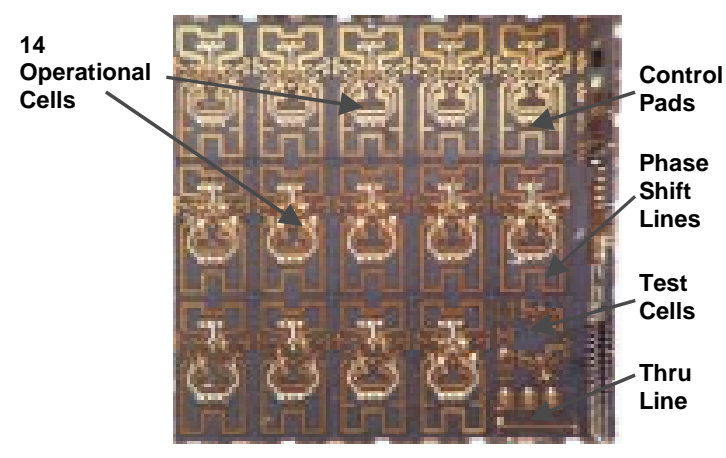

Fig. 7 Photograph of a phase shifter reticule with 142 bit phase shifters. 


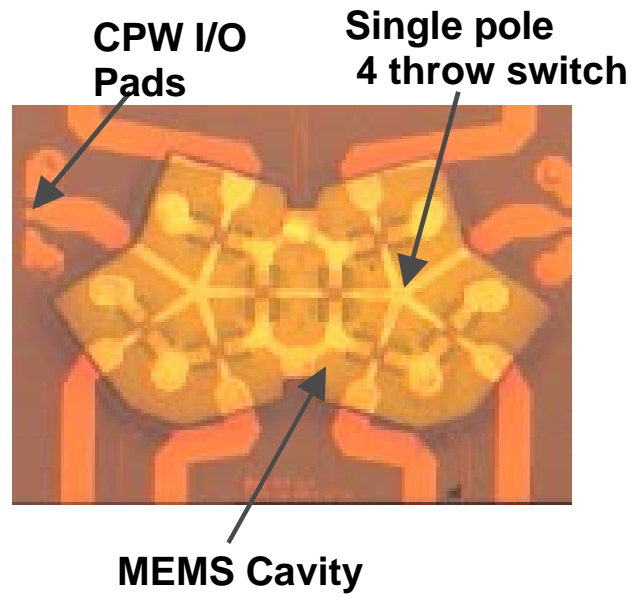

Fig. 8 Cavity in Chip-Scale-Package showing MEMS placement accuracy in cavity

\section{CONCLUSIONS}

RF MEMS Phase Shifters with low insertion loss, low power consumption, and reduced size and mass, promises significant communication system-level benefits to future space-based applications. System architectures that will meet these missions include phased arrays as well as conventional multi-beam antennas. These RF MEMS Phase shifters have been designed and fabricated with excellent performance and results. The overall size of the chip including the biasing network, making this the smallest RF MEMS phase shifter reported to-date.

\section{ACKNOWLEDGEMENT}

The authors would like to acknowledge the support of Dr. Larry Corey, Jackie Toussaint-Barker, Dr. John Smith, John Capone, John Logrando, and Bob Frankievich who supported the program.

\section{REFERENCES}

[1]. B. Pillans, S. Eshelman, A. Malczewski, J. Ehmke and C.L. Goldsmith, "Ka-Band RF MEMS phase shifters," IEEE Microwave Guided Wave Lett., vol. 9, pp. 520-522, Dec. 1999.

[2]. A. Malczewski, S. Eshelman, B. Pillans, J. Ehmke and C.L. Goldsmith, "X-Band RF MEMS phase shifters for phased array applications," IEEE Microwave Guided Wave Lett., vol. 9, pp. 517-519, Dec. 1999.

[3]. M. Kim, J.B. Hacker, R.E. Mihailovich, and J.F. DeNatale, "A DC-to-40 GHz four-bit RF MEMS truetime delay network," IEEE Microwave Wireless Comp. Lett., vol. 11, pp. 56-58, Feb. 2001.

[4]. J. S. Hayden, A. Malczewski, J. Kleber, C. L. Goldsmith, and G.M. Rebeiz, "2 and 4-Bit DC-18 GHz Microstrip MEMS Distributed Phase Shifters," 2001 IEEE MTT-S Intl. Microwave Symp. Dig., pp. 219-222, May 2001.

[5]. R. E. Mihailovich, M. Kim, J. B. Hacker, E. A. Sovero, J. Studer, J. A. Higgins and J. F. DeNatale, "MEM relay for reconfigurable RF circuits," IEEE Microwave Wireless Comp. Lett., vol. 11, no. 2, pp. 53-55, Feb 2001.

[6]. P. D. cooper, J.D. Kennedy, R. J. Street, W. P. Kornrumpf, W. M. Marcinkiewicz "Microwave High Density Interconnect (MHDI) - A Methodology for Batch Manufactured T/R Modules" Eight GaAs MAN Tech Conference, May, 1994

[7]. P. D. cooper, M. J. Fithian, R. J. Street, W. P. Kornrumpf, J. Santoro, “ A C-Band GaAs MMIC T/R Module using Microwave High Density Interconnect Technology", GOMAC - 92 proceedings, November, 1993

[8] A. Pham, J. Laskar, V. Krishnamurthy, H.S. Cole, and T. Sitnik-Nieters, "Ultra low loss millimeter wave MCM interconnects," Electrical Performance of Electronic Packaging, 1997, IEEE 6th Topical Meeting on, pp. 213-216, 1997.

[9], T. Gorczyca, Patent 6,255,137, "Method For Making Air Pockets In An HDI Context", July, 2001 